\title{
The galactic center: The ideal laboratory for studying supermassive black holes
}

\author{
Frank Eisenhauer
}

\author{
Max-Planck-Institut für extraterrestrische Physik, \\ Giessenbachstrasse, 85748 Garching, Germany, \\ email: eisenhau@mpe.mpg.de
}

\begin{abstract}
The Galactic Center constitutes the best astrophysical evidence for the existence of black holes, and it is the ideal laboratory for studying physics in the vicinity of such objects. The combination of infrared observations of three dimensional orbits of stars within the central light days and the extreme compactness and motionlessness of the radio-counterpart of the gravitational center have shown beyond any reasonable doubt that the Galactic Center harbors a supermassive black hole. The flaring activity from the black hole gives first insights to the physical processes close to the last stable orbit. Here I review the current state of observations and theory of the Galactic Center black hole and give an update on the latest results. I also outline the next steps towards even higher angular resolution observations, which give promise to directly probe the physics and space-time curvature just outside the event horizon.
\end{abstract}

Keywords. Galaxy: center, black hole physics, relativity, techniques: high angular resolution, interferometric

\section{References}

Backer, D. C. \& Sramek, R. A., 1999, ApJ, 524, 805

Balick, B. \& Brown, R. L., 1974, ApJ, 194, 265

Bower, G. C., Falcke, H., Herrnstein, R. M., Zhao, J.-H., Goss, W. M., \& Backer, D. C., 2004, Sci, 304, 704

Bower, G. C., Goss, W. M., Falcke, H., Backer, D. C., \& Lithwick, Y., 2006, ApJ, 648, L127

Broderick, A. E., Fish, V. L., Doeleman, S. S., \& Loeb A., 2009, ApJ, 697, 45

Broderick, A. E. \& Loeb, A., 2005, MNRAS, 363, 353

Broderick, A. E., Loeb, A., \& Narayan, R., 2009, arXiv:0903.1105

Broderick, A. E. \& Narayan, R., 2006, ApJ, 638, L21

Chapline, G., 2005, arXiv:astro-ph/0503200

Do, T., Ghez, A. M., Morris, M. R., Yelda, S., Meyer, L., Lu, J. R., Hornstein, S. D., \& Matthews, K., 2009, ApJ, 691, 1021

Doeleman, S. S., et al., 2008, Natur, 455, 78

Eckart, A. \& Genzel, R., 1996, Natur, 383, 415

Eckart, A., Schödel, R., Meyer, L., Trippe, S., Ott, T., \& Genzel, R., 2006, A\&A, 455, 1

Eisenhauer, F., et al., 2005, ApJ, 628, 246

Eisenhauer, F., et al., 2008, SPIE, 7013, 69

Eisenhauer, F., Perrin, G., Rabien, S., Eckart, A., Lena, P., Genzel, R., Abuter, R., \& Paumard, T., 2005, AN, 326, 561

Falcke, H., Melia, F., \& Agol, E., 2000, ApJ, 528, L13

Fish, V. L., Broderick, A. E., Doeleman, S. S., \& Loeb, A., 2009, ApJ, 692, L14

Fragile, P. C. \& Mathews, G. J., 2000, ApJ, 542, 328

Fujii, Y., \& Maeda, K.-I., 2003, The Scalar-Tensor Theory of Gravitation, Cambridge University Press, ISBN 0521811597

Genzel, R., Hollenbach, D., \& Townes, C. H., 1994, RPPh, 57, 417 
Genzel, R., Schödel R., Ott, T., Eckart, A., Alexander, T., Lacombe, F., Rouan, D., \& Aschenbach, B., 2003, Natur, 425, 934

Ghez, A. M., Klein, B. L., Morris, M., \& Becklin, E. E., 1998, ApJ, 509, 678

Ghez, A. M., et al., 2008, ApJ, 689, 1044

Gillessen, S., Eisenhauer, F., Trippe, S., Alexander, T., Genzel, R., Martins, F., \& Ott, T., 2009, ApJ, 692, 1075

Hamaus, N., Paumard, T., Müller, T., Gillessen, S., Eisenhauer, F., Trippe, S., \& Genzel, R., 2009, ApJ, 692, 902

Huang, L., Cai, M., Shen, Z.-Q., \& Yuan, F., 2007, MNRAS, 379, 833

Jaroszynski, M., 1998, AcA, 48, 653

Lacy, J. H., Townes, C. H., Geballe, T. R., \& Hollenbach, D. J., 1980, ApJ, 241, 132

Levin, Y. \& Beloborodov, A. M., 2003, ApJ, 590, L33

Mazur, P. O. \& Mottola, E., 2001, arXiv:gr-qc/0109035

Meyer, L., Do, T., Ghez, A., Morris, M. R., Yelda, S., Schödel, R., \& Eckart, A., 2009, ApJ, 694, L87

Narayan, R., Garcia, M. R., \& McClintock, J. E., 1997, ApJ, 478, L79

Paumard, T., et al., 2005, AN, 326, 568

Psaltis, D., 2004, AIPC, 714, 29

Reid, M. J. \& Brunthaler, A., 2004, ApJ, 616, 872

Reid, M. J., Readhead, A. C. S., Vermeulen, R. C., \& Treuhaft, R. N., 1999, ApJ, 524, 816

Rubilar, G. F. \& Eckart, A., 2001, A\&A, 374, 95

Shen, Z.-Q., Lo, K. Y., Liang, M.-C., Ho P. T. P., \& Zhao, J.-H., 2005, Natur, 438, 62

Townes, C. H., Lacy, J. H., Geballe, T. R., \& Hollenbach, D. J., 1983, Natur, 301, 661

Trippe, S., Paumard, T., Ott, T., Gillessen, S., Eisenhauer, F., Martins, F., \& Genzel R., 2007, MNRAS, 375, 764

Weinberg, N. N., Milosavljević M., \& Ghez, A. M., 2005, ApJ, 622, 878

Will, C. M., 2008, ApJ, 674, L25

Wollman, E. R., Geballe, T. R., Lacy, J. H., Townes, C. H., \& Rank, D. M., 1977, ApJ, 218, L103

Yuan, F., Quataert, E., \& Narayan, R., 2004, ApJ, 606, 894

Zucker, S., Alexander, T., Gillessen, S., Eisenhauer, F., \& Genzel, R., 2006, ApJ, 639, L21 